\title{
Time course reconstruction of fetal cardiac signals from $f$ MCG: independent component analysis versus adaptive maternal beat subtraction
}

\author{
S Comani ${ }^{1,2}$, D Mantini ${ }^{3}$, A Lagatta ${ }^{2}$, F Esposito ${ }^{4}$, S Di Luzio ${ }^{1,2}$ \\ and G L Romani ${ }^{1,2}$ \\ ${ }^{1}$ Department of Clinical Sciences and Bio-Imaging, Chieti University, Chieti, Italy \\ 2 ITAB-Institute of Advanced Biomedical Technologies, University Foundation 'G D'Annunzio', \\ Chieti University, Chieti, Italy \\ ${ }^{3}$ Department of Informatics and Automation Engineering, Marche Polytechnic University, \\ Ancona, Italy \\ ${ }^{4}$ Second Division of Neurology, Second University of Naples, Naples, Italy \\ E-mail: comani@itab.unich.it
}

Received 13 April 2004, accepted for publication 7 July 2004

Published 11 August 2004

Online at stacks.iop.org/PM/25/1305

doi:10.1088/0967-3334/25/5/019

\begin{abstract}
M-mode and pulsed Doppler echocardiography, cardiotocography and transabdominal fetal ECG are available in clinical practice to monitor fetal cardiac activity during advancing gestation, but none of these methods allows the direct measurement of morphological and temporal parameters for fetal rhythm assessment. Fetal magnetocardiograms (fMCGs) are noninvasive recordings of magnetic field variations associated with electrical activity of the fetal heart obtained with superconducting sensors positioned over the maternal abdomen inside a shielded room. Because of maternal cardiac activity, fMCGs are contaminated by maternal components that need to be eliminated to reconstruct fetal cardiac traces. The aim of the present work was to use two methods working in the time domain, an independent component analysis algorithm (FastICA) and an adaptive maternal beat subtraction technique (AMBS), for the retrieval of fetal cardiac signals from fMCGs. Detection rates of both methods were calculated, and FastICA and AMBS performances were compared in the context of clinical applications by estimating several temporal and morphological characteristics of the retrieved fetal traces, such as the shape and duration P-QRS-T waves, arrhythmic beat detection and classification, and noise reduction. Quantitative and qualitative comparison produced figures that always suggested that FastICA was superior to AMBS from the perspective of clinical use of the recovered fetal signals.
\end{abstract}


Keywords: fetal magnetocardiography, ICA, signal processing, electrophysiological measurements, prenatal diagnosis

\section{Introduction}

Monitoring fetal cardiac electrical activity during advancing gestation is of primary importance for the assessment of morphological and temporal parameters useful for an early and accurate diagnosis of fetal rhythm disturbances and possible medical treatment. Fetal arrhythmias hold an increased risk of cardiac malformation (Kleinman et al 1983), and although single extrasystoles, frequently encountered during pregnancy, spontaneously disappear before or after birth, a chance of about $1-2 \%$ exists that isolated extrasystoles progress to supraventricular tachycardias, which are the most common form of life-threatening fetal arrhythmia (Chaoui et al 1991, Wakai et al 2003). Therefore, an early assessment of the fetal cardiac rhythm is recommended, but, at present, no reliable technique allowing a direct measure and evaluation of fetal electrophysiological signals is available in clinical practice (van Leeuwen et al 1999, Peters et al 2001, Lewis 2003).

Among the noninvasive methods accessible for antenatal monitoring of fetal cardiac activity, cardiotocography (CTG), which is based on pressure changes measured on the maternal abdomen, only provides information on the fetal heart rate (FHR) and does not allow registration of irregular beats (van Leeuwen et al 1999, Peters et al 2001). Moreover, trans-abdominal fetal electrocardiography (fECG) is often inadequate to describe the electrophysiology of the fetal heart, especially during the second half of pregnancy: in fact, the interfering effect of maternal cardiac activity and the insulating properties of vernix caseosa distort and lower the weak fetal signal preventing the recording of satisfactory traces (Peters et al 2001, Wakai et al 2000a, Taylor et al 2003). As a result, M-mode and pulsed Doppler fetal echocardiography (fECHO) is the method of choice, but it detects mechanical events following electrical myocardial activation, permitting only an indirect characterization of the electrophysiological activity of the fetal heart (Kleinman et al 1983, Peters et al 2001); moreover, fECHO is strongly dependent on the investigator's experience and on fetal position and movements (Fyfe et al 1993).

Magnetocardiography (MCG) is a noninvasive technique that allows the measurement of magnetic field variations associated with cardiac electrical activity by means of superconducting sensors (Della Penna et al 2000). Fetal magnetocardiography (fMCG) consists of MCG recordings above the abdomen of a pregnant woman that are a combination of signals produced by both the maternal and fetal hearts: in fact, magnetic fields are almost unaffected by the insulating properties of vernix caseosa, and $\mathrm{MCG}$ can therefore monitor the fetal cardiac activity during the prenatal period (Peters et al 2001, Stinstra and Peters 2002). FMCG traces are morphologically similar to ECG, and are characterized by a signalto-noise ratio (SNR) that increases with gestation (Peters et al 2001); the clinical usefulness of fMCG has already been demonstrated in a number of studies (Wakai et al 2000b, 2003, Menendez et al 2000, Khaler et al 2001, Horigome et al 2001, Stinstra et al 2002, Grimm et al 2003, Lowery et al 2003, Kandori et al 2003, Comani et al 2004a, 2004c).

However, a condition that has to be fulfilled for the trustworthy employment of fMCG is that fetal signals can be separated from maternal ones, which can be considered a noise component in the case of fetal applications. Reliable mathematical techniques are therefore necessary to obtain accurate reconstructions of the time course of fetal cardiac activity for a quantitative assessment of cardiac temporal and morphological parameters. 
In this paper, we present two methods, both working in the time domain and suitable for that purpose: (1) the fixed point ICA algorithm (FastICA), which is based on the independent component analysis (ICA) approach and has already been demonstrated to be an appropriate method for fMCG data processing (Comani et al 2004b, Mantini et al 2004); (2) the adaptive maternal beat subtraction (AMBS), which is a deterministic technique commonly used to recover fetal traces from fMCGs by iteratively eliminating the average maternal signal. The performances of FastICA and AMBS were compared with regard to the characteristics of the reconstructed fetal signals in the perspective of clinical applications.

\section{Materials and methods}

\subsection{Fetal MCG system and acquisition}

Fetal magnetocardiograms (fMCGs) are extremely weak signals having peak-to-peak intensity of a few pT; they were acquired with a 77-channel system whose sensors consisted of low-temperature dc-superconducting quantum interference device (dc-SQUID) integrated magnetometers able to measure magnetic field variations ranging from $0.01 \mathrm{pT}$ to $100 \mathrm{pT}$. Overall white noise density sensed by a dc-SQUID magnetometer was about $5 \mathrm{fT} \mathrm{Hz}^{-1 / 2}$ above $60 \mathrm{~Hz}$ (Della Penna et al 2000). The 77 magnetometers, kept at $4 \mathrm{~K}$ with a bath of liquid helium inside a cylindrical cryostat, were grouped into two sets: the first was composed of 55 measuring magnetometers, arranged at fixed distances $(32 \mathrm{~mm})$ on a plane close to the cryostat bottom and covering a circular area of $23 \mathrm{~cm}$ diameter; the second set included the remaining 22 magnetometers, positioned on higher planes and used for residual noise reduction. The planar geometry of the system was particularly convenient for measurements over the chest and abdomen; it worked in a magnetically shielded room to reduce the interference from environmental magnetic fields.

FMCGs were acquired from 18 volunteers longitudinally during pregnancy. Each volunteer signed a written informed consent before undergoing $\mathrm{fMCG}$ examination, which has been approved by the Ethics Committee of our university. All volunteers presented no cardiac disease and a normal pregnancy. FMCG was performed after morphological and functional evaluation of the fetal heart by means of M-2D pulsed color Doppler echocardiogram, which also provided information for MCG system positioning above the maternal abdomen.

Each acquisition lasted $5 \mathrm{~min}$ on average and provided a set of 55 simultaneous fMCG traces; sampling frequency was $10 \mathrm{kHz}$, converted into digital form with a resolution of 8 bits and digitally filtered between $0.016 \mathrm{~Hz}$ and $250.0 \mathrm{~Hz}$. Data were decimated in order to reduce the total amount of data, with a resulting sampling frequency of $1 \mathrm{kHz}$. A maternal electrocardiogram (ECG) was simultaneously acquired with three non-magnetic leads positioned on the mother's chest along orientations parallel to the $X-, Y$-, $Z$-axes of the internal reference system of the MCG device.

\subsection{Adaptive maternal beat subtraction $(A M B S)$ method}

Adaptive maternal beat subtraction (AMBS) is a deterministic technique used either to remove artifacts with a known morphology or to recover selected signals from a noisy registration. In the case of physiological quantities that need to be removed from recorded signals and that are characterized by a periodicity, their average behavior can be defined, correlated with the original recording and subtracted with an iterative procedure. Maternal cardiac activity, unavoidably recorded during fMCG, represents the major artifact to be eliminated when reconstructing fetal cardiac signals. 
The first step of AMBS is the identification of peaks during ventricular depolarization in the maternal heart, as recorded in fMCG traces. The wave corresponding to the depolarization of ventricles is known as QRS complex, and the R wave identifies the peak in the electrophysiological activity during a cardiac cycle. In order to recognize maternal $\mathrm{R}$ peaks in fMCG, maternal ECG traces are generally employed, because they usually have welldefined waveforms and contain negligible fetal signal components. As an alternative, fMCG recordings with high SNR can be used for the same purpose, but in this case a proper threshold must be chosen to categorize only maternal QRS complexes. The instants corresponding to the selected $\mathrm{R}$ peaks will be the trigger points used in all 55 raw fMCG traces to set the time window for the alignment and averaging of maternal beats (Abraham-Fuchs et al 1990).

In order to define a trigger point as the instant $t_{i}$ corresponding to the $\mathrm{R}$ wave peak in the $i$ th maternal beat, the quantity $Q(t)$ is calculated from the measured electrical potentials $V(t)$ according to

$$
Q(t)=\left|V(t) \frac{\mathrm{d} V(t)}{\mathrm{d} t}\right|
$$

Maximizing $Q(t)$ in a preset moving window, generally equal to $500 \mathrm{~ms}$, a sequence of trigger points is found and maternal QRS complexes are categorized. Trigger points are defined as the $t_{i}$ values corresponding to maximal $Q(t)$, with $i=\left[1,2, \ldots, N_{\text {beats }}\right]$.

Given the recorded fMCG trace $b(t)$, a subset of maternal beats exhibiting a similar duration at $98 \%$ confidence level is selected and used to calculate the averaged maternal beat. After calculating the average $\mathrm{RR}$ interval duration $T$, the values $T_{\text {pre }}=5 T / 12$ and $T_{\text {post }}=$ $7 T / 12$ are used as pre- and post-trigger, respectively, in order to define the extent and alignment of the time window.

Assuming that the number of selected maternal beats is $N_{\text {beats }}, B(\tau)$ is given by

$$
B(\tau)=\frac{\sum_{i=1}^{N_{\text {beats }}} b_{i}(\tau)}{N_{\text {beats }}}
$$

for $\tau=t_{i}+t$ and $t=-T_{\text {pre }}, \ldots,-1,0,1, \ldots, T_{\text {post }} ; N_{\text {beats }}>100$ is required to obtain a well-defined average maternal beat.

Since the time window is set equal to the maternal beat period, fetal signal components are randomly mixed in the averaging process and therefore suppressed.

The maternal trace is reconstructed from the averaged maternal beat using weights $c_{i}$ that are based on mutual correlation maximization:

$$
c_{i}=\frac{\sum_{t=-T_{\mathrm{pre}}}^{T_{\mathrm{post}}} B(\tau) \cdot b\left(t_{i}+\tau\right)}{\sum_{t=-T_{\mathrm{pre}}}^{T_{\mathrm{post}}}|B(\tau)|^{2}}
$$

where $i=1,2, \ldots, N_{\text {beats. }}$ The reconstructed maternal trace $m(t)$ is given by

$$
m(t)=\sum_{i=1}^{N_{\text {beats }}} c_{i} B\left(\tau+t_{i}\right) .
$$

Finally, fetal traces are retrieved by subtracting $m(t)$ from fMCG recordings (Abraham-Fuchs et al 1991). AMBS is an adaptive procedure since it provides a different weight for every period in accordance with equation (3).

\subsection{ICA approach}

Independent component analysis (ICA) is a statistical digital signal processing (DSP) technique for separating a combined data set into independent components (Comon 1994, Bell and Sejnovsky 1995). 
Given a set of observations of $n$ random variables $\left[x_{1}(t), x_{2}(t), \ldots, x_{n}(t)\right]$, where $t$ is time, let us assume that a linear instantaneous mixture of $m$ independent components $\left[s_{1}(t), s_{2}(t), \ldots, s_{m}(t)\right]$ generated them. Therefore, we can state that

$$
\left[\begin{array}{l}
x_{1}(t) \\
x_{2}(t) \\
\cdots \\
\cdots \\
x_{n}(t)
\end{array}\right]=A\left[\begin{array}{l}
s_{1}(t) \\
s_{2}(t) \\
\cdots \\
s_{m}(t)
\end{array}\right]
$$

where $A=\left[a_{1}, \ldots, a_{m}\right]$ is a constant full-rank $[n \times m]$ matrix called the mixing matrix. Since background noise $n(t)$ is generally present in all fMCG measurements, the ICA model given in (5) should be extended to $x(t)=A s(t)+n(t)$. Anyhow, noise can be considered an independent signal having a Gaussian distribution. In order to accomplish source separation, model (5) was assumed to have at most one $s_{i}(t)$ with Gaussian distribution, which corresponds to background noise (Fiori 2003).

ICA consists in estimating the matrix $A$ and the sources $s_{i}(t)$ from the observed $x_{j}(t)$. Being ICA based on neural networks, the ICA problem can be solved under the condition that the number of observations $(n)$ is greater than or equal to the number of independent components $(m)$. Typical ICA methods estimate an un-mixing matrix $W$ such as

$$
\left[\begin{array}{l}
s_{1}(t) \\
s_{2}(t) \\
\cdots \\
s_{m}(t)
\end{array}\right]=W\left[\begin{array}{l}
x_{1}(t) \\
x_{2}(t) \\
\cdots \\
\cdots \\
x_{n}(t)
\end{array}\right]
$$

under the condition that $s_{i}(t)$ be as independent as possible, for $i=1, \ldots, m$.

The basic hypothesis to be satisfied in order to apply ICA to fMCGs is that fMCGs must represent a mixture of statistically independent signals produced by separated sources. As already stated, one source is noise, while fMCG traces contain other two independent signals originating from physically distinct sources, the maternal and fetal hearts. The statistical independence of simultaneous maternal and fetal signals was verified by calculating mutual information (MI), which measures the distance between their probability distribution functions; since MI values for maternal and fetal signals were always around zero, the ICA fundamental constraint was satisfied (Comani et al 2004b).

2.3.1. Preprocessing for ICA. In order to obtain $A$ by estimating a minimum number of parameters it is necessary to center and whiten the acquired data (Hyvärinen and Oja 1997). Centering is performed by subtracting the $j$ th average value from each mixed signal $x_{j}(t)$, so that the vector of independent components $s_{i}(t)$ becomes zero mean.

Whitening is a linear transformation of vector $x(t)$ into another vector $\bar{x}(t)$ whose components are uncorrelated and with variances equal to unity. A common method for whitening is the eigenvalue decomposition (EVD) of the covariance matrix $E\left\{x x^{\mathrm{T}}\right\}=E D E^{\mathrm{T}}$, where $E$ is the orthogonal matrix of eigenvectors of $E\left\{x x^{\mathrm{T}}\right\}$ and $D$ is the diagonal matrix $D=\operatorname{diag}\left(d_{1}, d_{2}, \ldots, d_{n}\right)$ of its eigenvalues (Yang and Wang 1999).

The whitened vector $\bar{x}$ can be calculated as

$$
\bar{x}=E D^{-1 / 2} E^{\mathrm{T}} x
$$

with $D^{-1 / 2}=\operatorname{diag}\left(d_{1}^{-1 / 2}, d_{2}^{-1 / 2}, \ldots, d_{n}^{-1 / 2}\right)$. Whitening transforms the mixing matrix $A$ into a new matrix $\bar{A}$. Therefore,

$$
\bar{x}=E D^{-1 / 2} E^{\mathrm{T}} A s=\bar{A} s
$$


where $\bar{A}$ is orthogonal and allows minimizing of the number of parameters to be estimated. Mixing matrix $A$ can be computed as

$$
A=E D^{1 / 2} E^{\mathrm{T}} \bar{A} .
$$

2.3.2. FastICA: theory and algorithm. In order to solve the ICA problem concerning fMCGs, the fixed point ICA algorithm (FastICA) was chosen because it is a neural algorithm particularly efficient and light from the point of view of computational effort (Comani et al 2004b, Hyvärinen 1999).

In order to estimate the independent sources $s_{i}(t)$ this framework uses the principle that signal components forced to be mutually statistically independent tend to be characterized by probability distributions that are not Gaussian. Starting from the definition of a single independent component:

$$
s_{i}(t)=w^{\mathrm{T}} x=\sum_{j=1}^{n} w_{j} x_{j}
$$

FastICA uses the method of the kurtosis (fourth-order cumulant) of the signals (Hyvärinen and Oja 1997, Hyvärinen 1999, Cardoso 1992), which is defined for a zero-mean random variable $v$ as

$$
\operatorname{kurt}(v)=E\left\{v^{4}\right\}-3\left(E\left\{v^{2}\right\}\right)^{2} .
$$

Kurtosis is null for a Gaussian random variable, it is positive for densities peaked at zero and negative for flatter densities. This means that kurtosis is suitable to assess the statistical independence of given variables.

In order to maximize and/or minimize the kurtosis under the constraint $\|w\|=1$, neural algorithms can be used (Bell and Sejnovsky 1995, Cardoso 1997, Hyvärinen and Oja 2000). Using the natural gradient method, the neural network will have the following learning rule:

$w(t+1)=w(t) \pm \mu(t)\left[\bar{x}(t)\left(w(t)^{\mathrm{T}} \bar{x}(t)\right)^{3}-3\|w(t)\|^{2} w(t)+f\left(\|w(t)\|^{2}\right) w(t)\right]$

where $\bar{x}(t)$ is the sequence of whitened signals, $\mu(t)$ is the learning rate and $f$ is a penalty term due to the constraint $\|w\|=1$.

The learning rule will stop at a fixed point for which $\left|w^{\mathrm{T}}(t) w(t-1)\right|$ is sufficiently close to unity. The linear combination $w^{\mathrm{T}} x$ will be one of the required independent components in accordance with equation (10).

The FastICA algorithm is derived from the described theory (Hyvärinen and Oja 1997, Hyvärinen 1999); it consists of (1) randomly choosing an initial vector $w(0)$ with unit norm, (2) applying the fixed-point iteration rule $w(t)=E\left\{x\left(w(t-1)^{\mathrm{T}} x\right)^{3}\right\}-3 w(t-1)$ to approximate $w(t)$, (3) normalizing $w(t)$ and (4) repeating points (2) and (3) until $\left|w^{\mathrm{T}}(t) w(t-1)\right|$ is sufficiently close to unity. One ICA basis vector will then be estimated. Other ICA basis vectors can be estimated by sequentially projecting a new starting basis vector $w(0)$ onto the subspace orthogonal to the one covered by the previous vectors (Comani et al 2004b).

2.3.3. Application of FastICA to fMCG. Before being used as input traces for FastICA, the $55 \mathrm{fMCGs}$ were grouped in subsets, filtered between 0.4 and $150 \mathrm{~Hz}$ and reduced by a factor of 3 .

FastICA iterations were set to produce six independent components, with no predefined order and random amplitude and polarity (Fiori 2003). Thus, independent components related to fetal cardiac activity were selected on the basis of heart rate (always higher for the fetus as compared to the mother in a ratio of approximately $3: 2$ ). Those components were compared 
with original fMCG recordings in order to restore the correct polarity by multiplying each component either by 1 or by -1 ; finally, they were summed to reconstruct a comprehensive fetal signal. The appropriate signal intensity was also retrieved collecting the necessary information from raw fMCG, and the correct time scale was recovered by interpolation (Comani et al 2004b).

\subsection{Post-processing}

In general, both FastICA and AMBS allowed the reconstruction of fetal traces, although in some cases the original recordings had extremely weak fetal QRS complexes, with the same order of magnitude of background noise. Consequently, the reconstructed traces might be affected by a low SNR that could be enhanced by using a smoothing procedure based on a Kaiser filter (Mitra and Kaiser 1993) working with a moving window. The smoothing factor $\sigma$, defined as the number of samples in the moving window, was expressed in milliseconds and was set inversely proportional to the signal amplitude in the time interval under investigation. Therefore, a typical $\sigma$ value of $50 \mathrm{~ms}$ was used for $\mathrm{P}$ and T waves, which are slow cardiac events characterized by low signal intensity, and therefore were more affected by noise; conversely, a smoothing factor of $12 \mathrm{~ms}$ was used for rapid occurrences such as QRS complexes, which remained essentially unmodified.

\section{Results}

\subsection{Characteristics of acquired fMCG}

Eighteen pregnant women underwent recurring fMCG acquisitions every 3-4 weeks starting from about 22 weeks' gestation until delivery for a total of 2-4 sessions each, depending on pregnancy evolution. A total of $51 \mathrm{fMCG}$ data sets of 55 recordings each were available for the reconstruction of fetal signals in different gestational epochs.

Although acquired in a shielded room, raw fMCGs could still be affected by several sources of noise, such as fetal movements, which might generate both slow and very rapid baseline shifts, or white noise and residual $50 \mathrm{~Hz}$ contamination. The resulting SNR, calculated with respect to fetal QRS complexes, could even be less than unity. Other factors might affect the SNR of acquired traces, such as early gestational age, which implied smaller fetal signals, and unfavorable fetal position or maternal obesity that could further increase the distance between the fetal heart and the sensor plane.

\subsection{Effectiveness of FastICA and AMBS}

The efficiency of FastICA and AMBS was evaluated for all acquired fMCGs, hence including a variety of boundary conditions more or less favorable to a clear fetal signal reconstruction. FastICA and AMBS were compared with respect to morphological and temporal aspects of an electrophysiological cardiac trace. In particular, we assessed their capability of (1) detecting fetal QRS complexes; (2) reconstructing fetal P-QRS-T waves preserving unmodified waveforms and durations; (3) detecting and reconstructing fetal cycles in the case of simultaneous maternal and fetal beats; (4) detecting fetal arrhythmias and (5) reducing all types of noise and increasing the SNR of reconstructed traces.

3.2.1. QRS detection rates. The rates of success of FastICA and AMBS in detecting fetal QRS complexes out of the recorded signals were calculated for all data sets; the results are 
Table 1. FastICA and AMBS detection rates expressed as a function of gestational period, given in weeks; values are given as the ratio between the number of reconstructed fetal traces and the number of available fMCG data sets; per cent values are given in parentheses.

\begin{tabular}{llllll}
\hline & \multicolumn{4}{c}{ Gestational periods (weeks) } \\
\cline { 2 - 6 } & $22-25$ & $26-29$ & $30-33$ & $34-37$ & Total (22-37) \\
\hline Available raw fMCG data sets & 10 & 15 & 13 & 13 & 51 \\
FastICA: QRS detection rate $^{\mathrm{a}}$ & $8 / 10(80)$ & $14 / 15(93)$ & $13 / 13(100)$ & $13 / 13(100)$ & $48 / 51(94)$ \\
AMBS: QRS detection rate $^{\mathrm{a}}$ & $3 / 10(30)$ & $10 / 15(67)$ & $10 / 13(77)$ & $11 / 13(85)$ & $34 / 51(67)$ \\
FastICA: P-QRS-T detection rate $^{\mathrm{b}}$ & $7 / 10(78)$ & $13 / 15(85)$ & $13 / 13(100)$ & $13 / 13(100)$ & $46 / 51(90)$ \\
AMBS: $\mathrm{P}-\mathrm{QRS}-\mathrm{T}$ detection rate $^{\mathrm{b}}$ & $0 / 10(0)$ & $4 / 15(27)$ & $6 / 13(46)$ & $9 / 13(69)$ & $19 / 51(37)$ \\
\hline
\end{tabular}

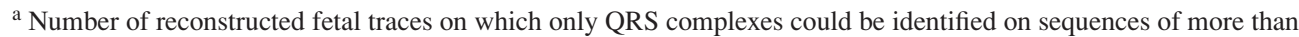
25 cardiac cycles.

b Number of reconstructed fetal traces on which all P-QRS-T waves could be clearly identified on sequences of more than 25 cardiac cycles.

given in table 1 as a function of gestational age. Detection rates were calculated relative to the total number of available fMCG data sets for each gestational period; the overall detection rates obtained for FastICA (94\%) and AMBS (67\%) were, respectively, better and worse than those given in the literature, included between $80 \%$ and $85 \%$ (Horigome et al 2000, Kaler et al 2002).

3.2.2. P-QRS-T waves detection rates. The outcome changed drastically when analyzing the effectiveness of FastICA and AMBS in the reconstruction of fetal signals where the onset and offset of all cardiac waves could be doubtlessly identified for a reliable measurement of cardiac time intervals. In fact, it was possible to clearly categorize P-QRS-T waves on several sequences of more than 25 consecutive beats on almost all traces reconstructed with FastICA, with an overall detection rate of 90\%; the figure obtained for the earliest gestational period (table 1) is particularly remarkable. On the other hand, the values obtained for AMBS were certainly worse, with a general detection rate of $37 \%$; figures were even worse for early gestation (table 1).

As regards the preservation of original shape and duration of cardiac waves, figure 1 shows an example of a raw fMCG recorded at 36 weeks' gestation (figure 1(a)) and the traces reconstructed from it by means of FastICA and AMBS (figures 1(b) and (d), respectively); the same fetal traces after smoothing are shown in figures 1(c) and (e), respectively. It is evident that FastICA retrieved $\mathrm{P}$ and $\mathrm{T}$ waves reproducible throughout the trace with no wave distortion, whereas AMBS was much less effective, even after smoothing. The correct timing of both methods with respect to $\mathrm{R}$ peaks can also be appreciated from figure 1 .

The effectiveness of FastICA is further demonstrated in figure 2, where the timing of onset and offset of fetal cardiac waves is clearly simultaneous in traces obtained from clusters opposite with respect to the fetal heart.

3.2.3. Simultaneous maternal and fetal beats. During a registration maternal QRS complexes often hid the weaker fetal beats because of different maternal and fetal heart rates. FastICA properly reconstructed $100 \%$ of hidden fetal beats, preserving correct QRS amplitude and duration; again, AMBS performance was definitely inferior, with a rate of success of about $15 \%$; QRS amplitude and duration were always altered (figure 1, fifth cardiac cycle). 

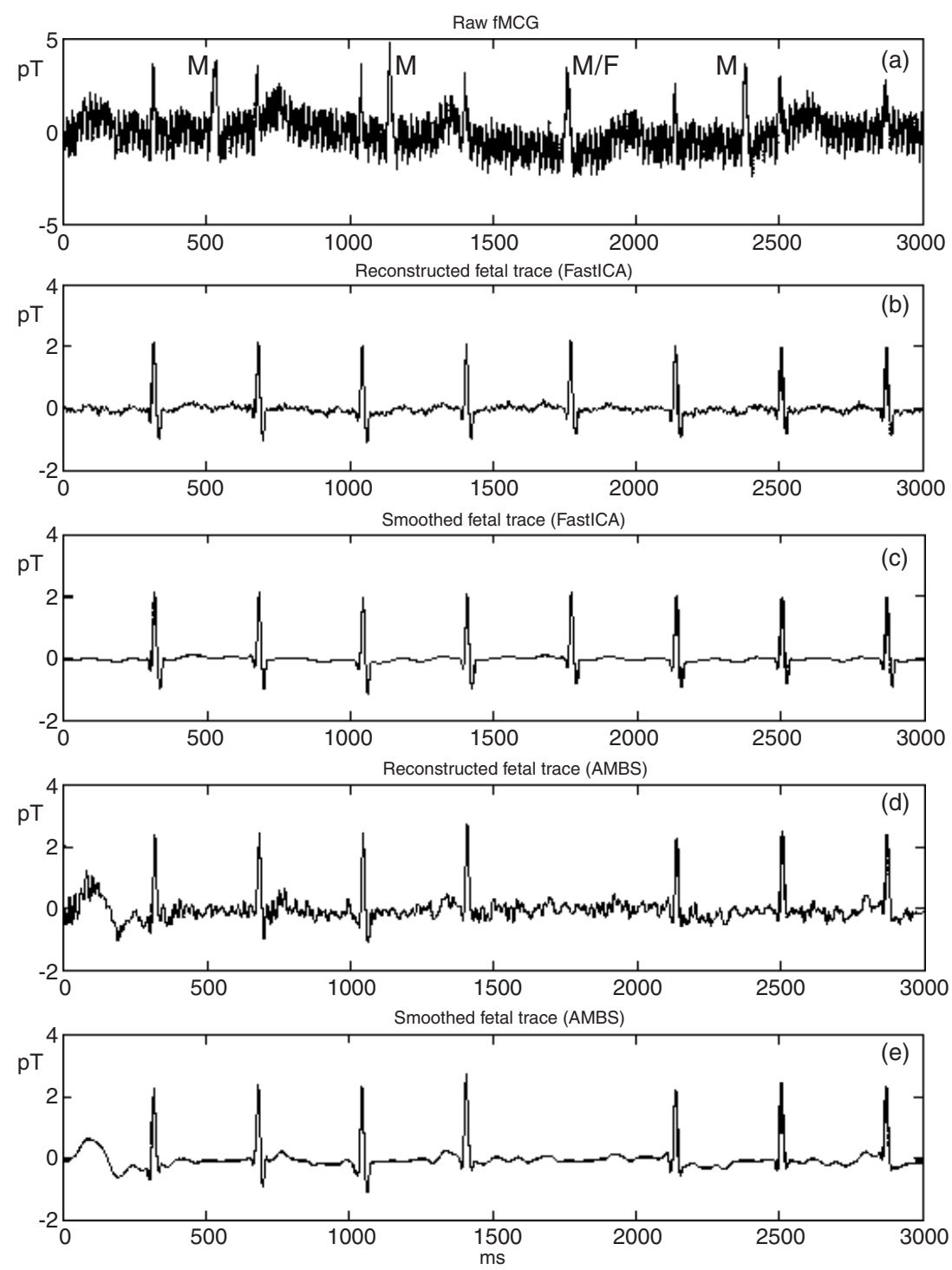

Figure 1. Original fMCG trace recorded at 36 weeks' gestation (a). Maternal QRS complexes are labeled with $\mathrm{M}$; the peak labeled M/F is a maternal QRS complex covering a fetal beat. The other peaks are fetal QRS complexes. The fetal signals reconstructed from trace (a) by means of FastICA (b) and smoothed (c), and obtained with AMBS (d) and smoothed (e) are shown. Time is given in milliseconds and signal intensity in pT.

3.2.4. Detection of fetal arrhythmias. The detection of fetal arrhythmias is a critical point in the perspective of clinical applications. Figure 3 shows a raw fMCG recorded at 37 weeks' gestation (figure 3(b)), which presents several difficulties for the reconstruction of a reliable fetal trace, such as low SNR, similar maternal and fetal QRS amplitude, baseline shift and fetal arrhythmias with a case of $\mathrm{R}$ wave polarity inversion. In order to permit an undisputed identification of maternal QRS complexes, the simultaneous maternal ECG is shown in figure 3(a). The fetal traces obtained with FastICA and AMBS are given in figures 3(c) and $(\mathrm{d})$, respectively. 


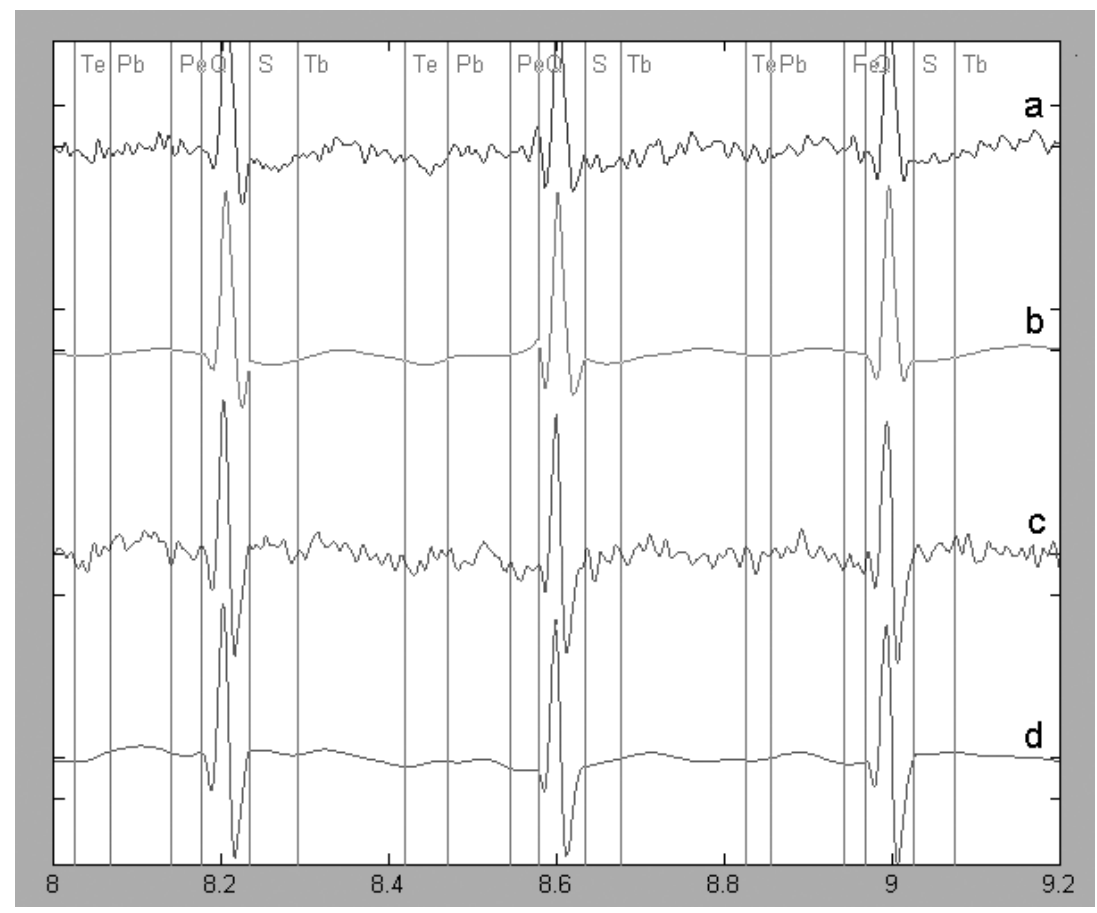

Figure 2. Fetal traces reconstructed with FastICA (a and c) using two opposite clusters of an fMCG data set recorded at 36 weeks' gestation; the same traces after smoothing are also shown (b and d). Onset and offset of P, QRS and T waves are identified with vertical lines on subsequent cardiac cycles to show simultaneity of timing in the reconstructed fetal signals. Time from the beginning of fMCG acquisition is given in seconds and signal intensity in arbitrary units.

Fetal extrasystolic beats (figures 3(c) and (d), second and fifth beats) were detected and correct QRS timing and polarity were retrieved by both methods. General rates of success for FastICA and AMBS were $100 \%$ and $85 \%$, respectively. FastICA also succeeded in restoring a trace of good quality, whereas traces obtained with AMBS were often affected by maternal interference, residual baseline shift and lower SNR (figure 3(d)).

A clear example of successful reconstruction of arrhythmia complexes with FastICA is given in figure 4. Traces obtained from a fMCG recorded at 36 weeks (figure 4(a)) by means of FastICA and AMBS are shown for comparison (figures 4(b) and (c)); the third and sixth cardiac cycles, corresponding to ectopic beats followed by a compensatory pause, are clearly visible in figure 4(b). Average sinus and arrhythmic beats, calculated on fetal cardiac cycles selected on the basis of ventricular frequency, were retrieved with FastICA (figures 4(d) and (e)) and AMBS (figures 4(f) and (g)); the superior performance of FastICA is evident from figure 4(e) relative to an averaged supraventricular extrasystole (SVE).

3.2.5. Noise reduction. FastICA and AMBS performances were also compared in cases of original fMCGs affected by low SNR and fetal QRS amplitude equivalent to background noise intensity, as in the example shown in figure 5(a), where fetal $\mathrm{R}$ peaks can hardly be distinguished. Traces reconstructed with FastICA generally displayed a clear cardiac signal; in figure 5(b) QRS complexes exactly corresponded to fetal R peaks visible in figure 5(a), and $\mathrm{P}$ and $\mathrm{T}$ waves were well defined. In contrast, traces obtained with AMBS usually detected 

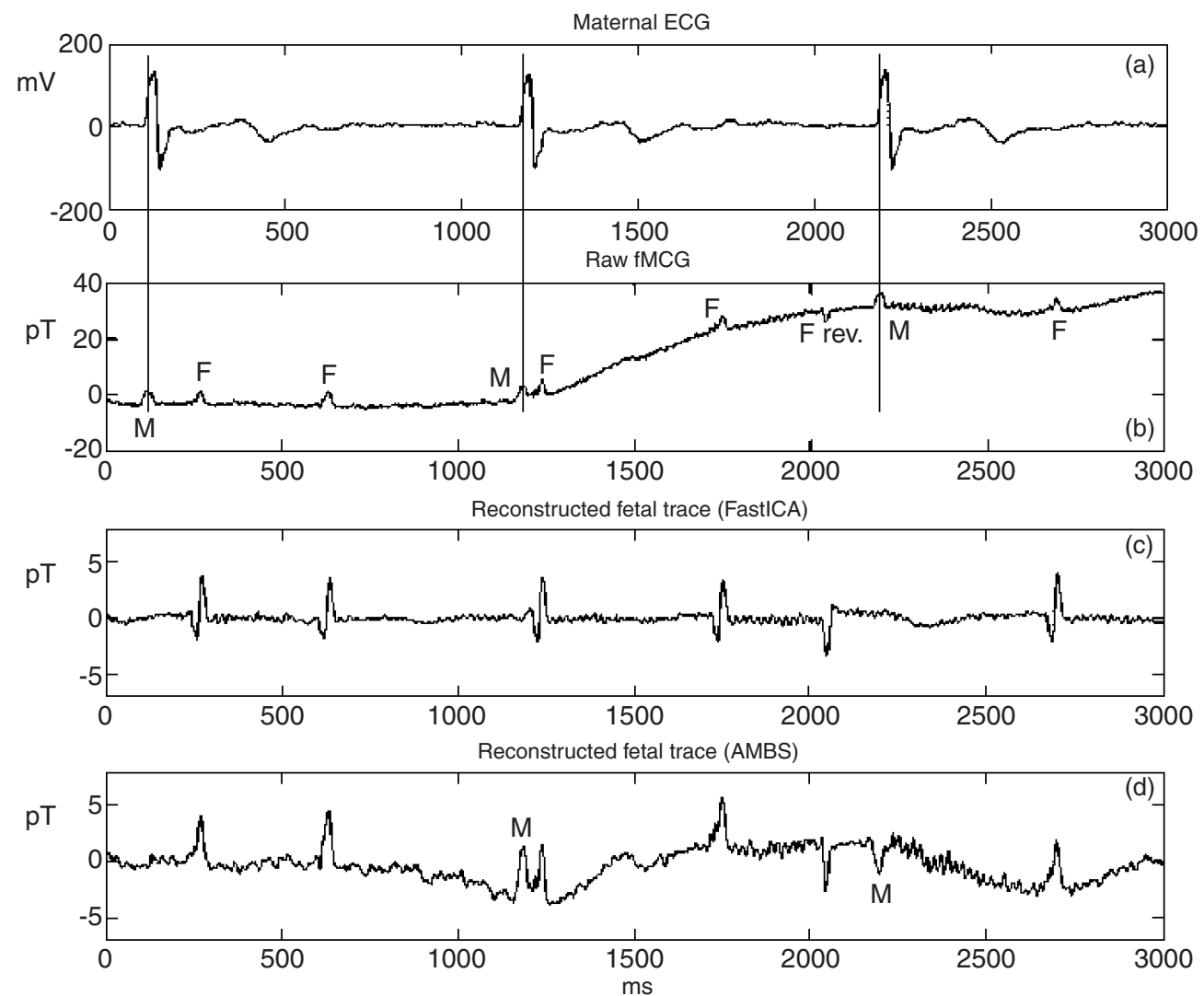

Figure 3. Original fMCG recorded at 37 weeks' gestation (b); maternal and fetal QRS complexes are labeled with $\mathrm{M}$ and $\mathrm{F}$, respectively; the peak labeled $\mathrm{F}$ rev. is a fetal QRS showing polarity inversion. The maternal ECG is given in (a) for certain identification of maternal QRS. The fetal signals reconstructed from trace (b) with FastICA (c) and AMBS (d) are shown. Time is given in milliseconds, ECG intensity in millivolts and magnetic signal intensity in pT.

only R peaks, as in figure 5(c) where no QRS characterization was possible because of bad waveform retrieval. The increased SNR is worth noting in figure 5(b) with respect to figure 5(c). The per cent noise reduction in the traces reconstructed with FastICA was $70 \%$ on average, whereas no noise decrease was attained with AMBS.

\section{Discussion and conclusions}

The effectiveness of a mathematical technique in reconstructing biological signals that are hidden in composite and noisy recordings strongly depends on trace quality. Fetal magnetocardiographic data may have an extremely variable SNR; it is a function of the characteristics of the acquisition system and whether it worked in a shielded room or not, but it also depends on fetal age, position, orientation, state and on the amount of white noise and residual magnetic contamination. All those factors participate to distort and cover the signals that need to be retrieved, and the final outcome of a recording session may as well provide traces that are fairly clear and stable, or blurred and unstable.

In general, the typical intensity of fetal cardiac activity is an order of magnitude lower than the maternal one; this means that signals coming from the fetal heart are extremely weak and 

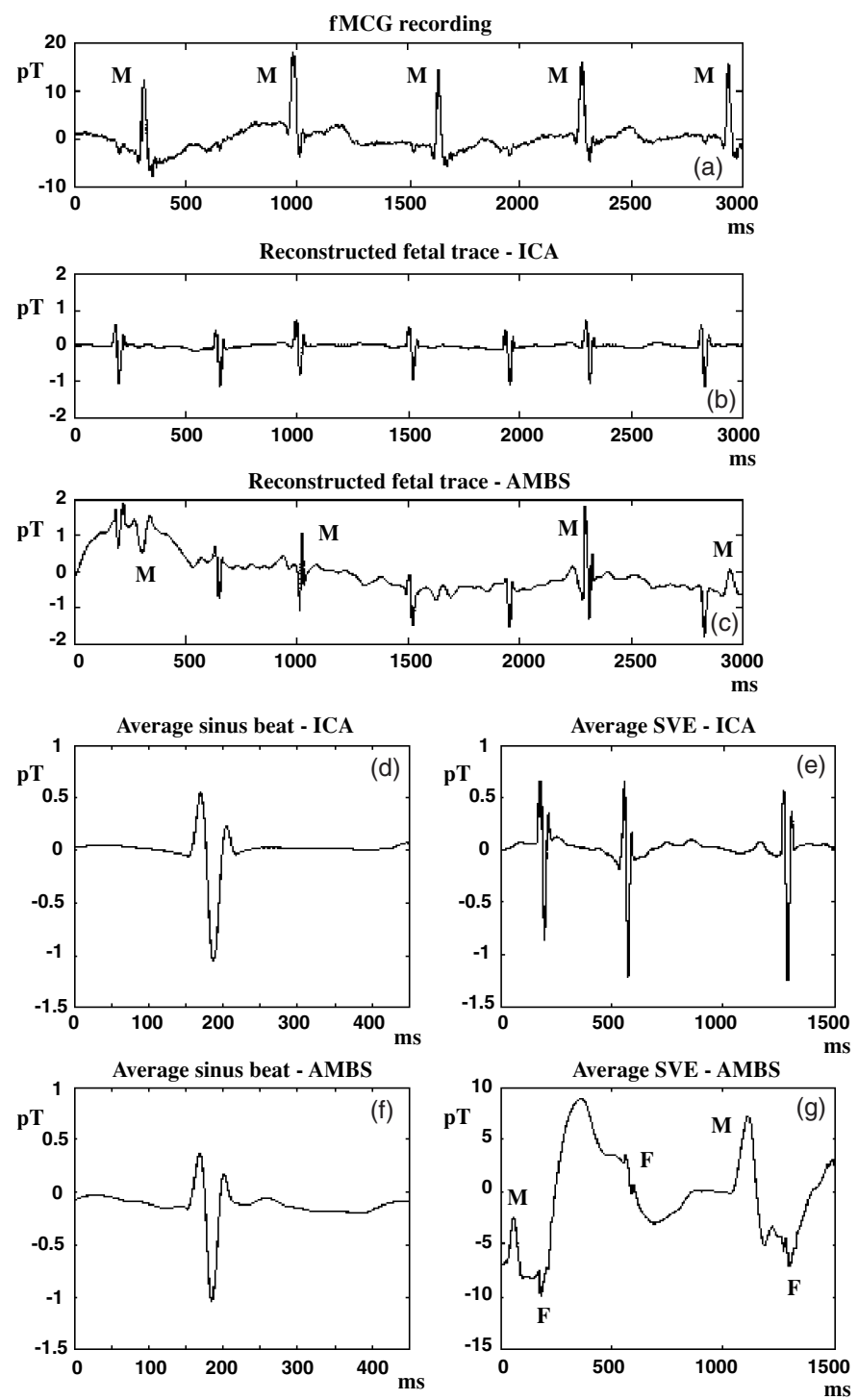

Figure 4. Example of fetal supraventricular extrasystoles with different coupling intervals; data refer to a pregnant woman at 36 weeks. The original fMCG recording (a), where fetal signals can hardly be identified, is shown; maternal QRS complexes are labeled with M. The corresponding fetal traces reconstructed with FastICA (b) and AMBS (c) are displayed in the figure. In (c) residual maternal R peaks are indicated with M. Supraventricular extrasystoles (3rd and 6th beats) with atrio-ventricular blocks, identified as pauses without a prematurity of the preceding QRS complex, are clearly detected in (b). Average sinus and supraventricular extrasystolic (SVE) beats are also given; they were calculated by selecting cardiac cycles with equal RR intervals from traces reconstructed with FastICA ( $\mathrm{d}$ and e) and AMBS (f and g). In (g) maternal R peak contamination, still present in the average beat, is labeled with $\mathrm{M}$, while fetal $\mathrm{R}$ peaks are indicated with F. Signal amplitudes are given in pT, time is measured in milliseconds. Average peak-to-peak amplitude of sinus fetal QRS complexes was about $1.5 \mathrm{pT}$ ((d) and (f)).

sometimes comparable to background noise, especially during early gestation. Occasionally, fetal beats, coincident with the maternal ones because of the relative heart rate of mother and fetus, are covered. 

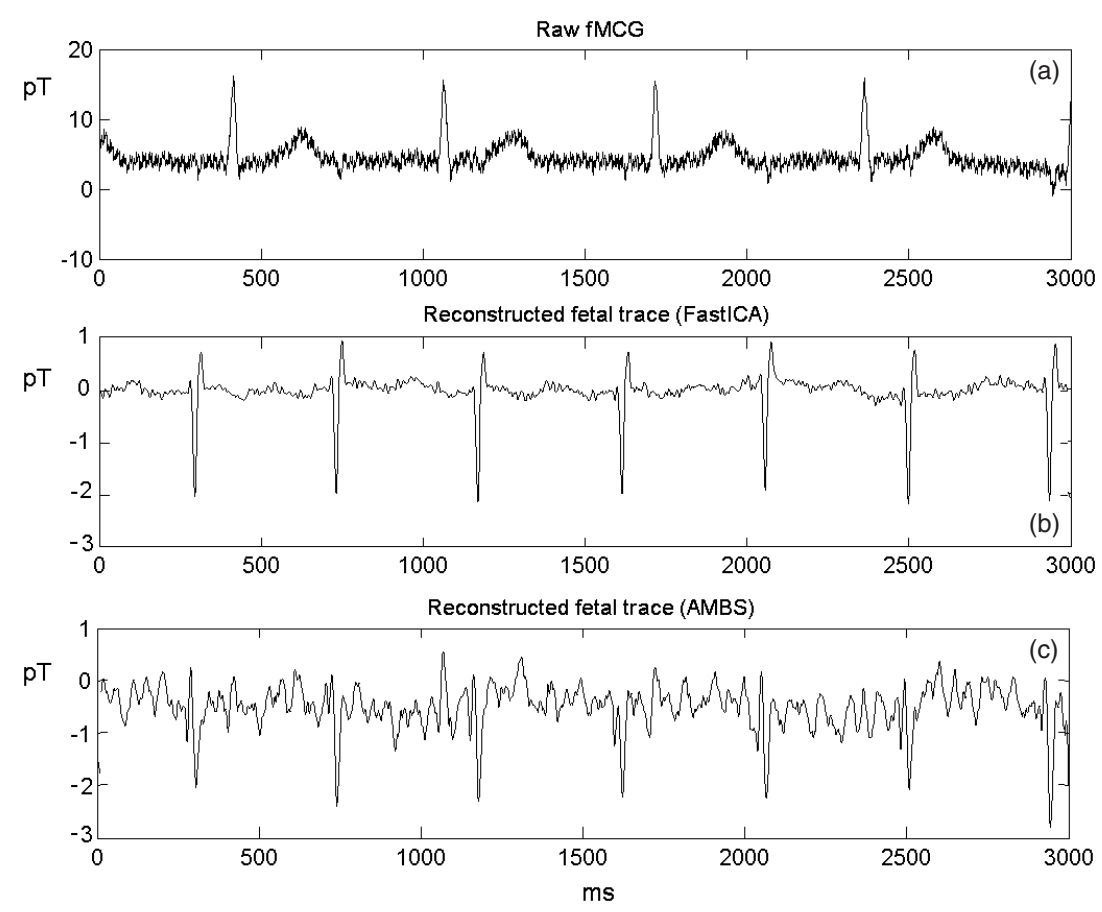

Figure 5. Example of fMCG recording with fetal signal amplitude of the same order of magnitude as of the background noise (a); fetal QRS complexes can hardly be distinguished, whereas evident QRS are only maternal. The fetal signals reconstructed from trace (a) with FastICA (b) and AMBS (c) are shown. Time is given in milliseconds and signal intensity in pT.

As regards the application of FastICA and AMBS to fMCG data, our results showed that both techniques were, in general, successful in reconstructing fetal cardiac signals, but their detection rates and performances were tremendously different, especially with respect to the quality of the reconstructed traces. In order to perform a comprehensive comparative study aiming at assessing the clinical usefulness of both techniques, $51 \mathrm{fMCG}$ data sets of 55 recordings each, acquired at different gestational ages and presenting various boundary conditions, were used.

In general, the effectiveness of both FastICA and AMBS enhanced with advancing gestation (table 1); this fact is certainly related to the mass of the fetal heart that increases on average by a factor of 12 from the 20th week of gestation to term, ranging from $1.8 \mathrm{~g}$ to $21.0 \mathrm{~g}$ (Peters et al 2001, St John Sutton et al 1984). The unquestionably small dimensions of the fetal heart also explain the low intensity of the associated magnetic signal, especially at early gestational epochs: peak-to-peak intensity throughout the second half of pregnancy ranges from 500 fT to 3 pT approximately.

Notwithstanding these real facts, the detection rates obtained with FastICA were definitely better than the figures reported in the literature (80-85\%), with an average score of $94 \%$ and a range between $80 \%$ and $100 \%$ from 22 weeks until end of pregnancy. Particularly remarkable is the value obtained for early gestational period with a failure in detecting QRS complexes only in 3 cases out of 51, all referring to epochs earlier than 26 weeks.

In contrast, AMBS was much less effective, with an average value of $67 \%$ and a range between $30 \%$ and $85 \%$ from 22 weeks to the final stages of pregnancy, hence failing to detect QRS complexes in a total of 17 cases out of 51. 
The figures obtained for the detection rate of all cardiac waves were even more meaningful from the clinical point of view. In fact, the quantification of RR intervals, obtainable from the timing of QRS peaks, is inadequate for the characterization of cardiac waveforms and intervals that are necessary for the diagnosis of a variety of cardiac rhythm disturbances (Wakai et al 2003, Stinstra et al 2002, Grimm et al 2003, Lowery et al 2003, Kandori et al 2003, Comani et al 2004a, 2004c). The P-QRS-T waves detection rate (table 1) was calculated by defining 'measurable' a reconstructed fetal trace in which the cardiac waves were sufficiently clear to permit the identification of their onset and offset on several sequences of more than 25 consecutive beats, hence allowing a reliable beat-to-beat estimate of all cardiac intervals (Comani et al 2004a). The general P-QRS-T waves detection rate of FastICA (90\%) continued to be high and still superior to the values reported in the literature; it was very good also for early epochs (78\%). Conversely, AMBS showed poor performance, ranging from $0 \%$ for early gestational periods to $69 \%$ towards the end of gestation, with an average figure of $37 \%$.

It should be underlined that the reference values reported in the literature considered the detection of QRS complexes on real time fetal signals, but the identification of $\mathrm{P}-\mathrm{QRS}-\mathrm{T}$ waves was performed on averaged fetal beats. In that perspective the results of FastICA were even more significant, because they permitted a reliable beat-to-beat quantification of all cardiac waves; the same was not possible on traces provided by AMBS, which needed to be averaged for a reliable $\mathrm{P}-\mathrm{QRS}-\mathrm{T}$ waves characterization.

The possibility of retrieving a consistent time course of fetal cardiac events prenatally and noninvasively is extremely valuable for a number of clinical applications (Wakai et al 2003, Stinstra et al 2002, Grimm et al 2003, Lowery et al 2003, Kandori et al 2003, Comani et al 2004a, 2004c). Aiming at assessing the reliability of reconstructed fetal traces in terms of a trustworthy representation of the underlying fetal cardiac electrophysiological activity, the validation of FastICA and its comparison to AMBS considered a number of morphological and temporal parameters.

As shown in figures 1 and 2, waveforms, intensities and durations of QRS complexes were preserved with both methods, also after smoothing; conversely, waveforms, intensities and durations of $\mathrm{P}$ and $\mathrm{T}$ waves were adequately reconstructed for all gestational periods only with FastICA; they were even more clearly defined after smoothing without any distortion.

In the case of overlapped maternal and fetal beats, FastICA provided a correct reconstruction of the hidden fetal beats in all cases, whereas AMBS only in $15 \%$. This is a critical point for the clinical use of $\mathrm{AMCG}$, because the reconstruction of a trace with missing beats might compromise a correct diagnosis even leading to inappropriate or unnecessary medical treatment.

The detection of fetal arrhythmias is also a decisive aspect of clinical application of fMCG. It requires not only a correct timing of cardiac events with a precision of a few milliseconds, but also reliable waveforms and signal polarities to characterize the arrhythmias. The detection rate of FastICA was again extremely good (100\%), and also AMBS performance was satisfactory $(85 \%)$; nonetheless, in many cases the traces reconstructed with AMBS were strongly affected by several sources of noise, such as residual maternal signal, unclear P and $\mathrm{T}$ waves definition and overall low SNR.

The presence of maternal components, although reduced, in the fetal traces obtained with AMBS is a consequence of the algorithm used; in fact, the maternal signal is eliminated from fMCGs by iteratively subtracting the average maternal beat from each cardiac cycle, which might have a slightly different morphology and intensity; this explains why small maternal $\mathrm{R}$ peaks can remain in the fetal trace. All those facts in general did not affect the retrieval of 
reliable average sinus beats, but prevented the recovery of average arrhythmic beats allowing dysrhythmia classification useful for clinical purposes.

In contrast, FastICA generally succeeded in reconstructing a reliable time course of cardiac activity also in arrhythmic fetuses, with clearly defined sinus and extrasystolic complexes. Although single extrasystoles are the most frequently encountered fetal arrhythmia and generally disappear during pregnancy or shortly after birth, isolated extrasystoles might progress to supraventricular tachycardias in about 1-2\% of cases (Chaoui et al 1991). Therefore, FastICA provided a powerful tool for the prenatal diagnosis and classification of electrophysiological dysfunctions; this feature is particularly valuable given the limits of other techniques recently applied for the noninvasive monitoring of fetal electrophysiology (Taylor et al 2003).

As regards background noise, no dumping effect was observed in traces reconstructed with AMBS, which is tailored to eliminate only the maternal signal from the recorded trace; therefore, a large percentage of noise present in fMCGs remains in the retrieved fetal traces, preventing a completely successful reconstruction of fetal cardiac waves and entailing the use of averaged fetal beats for clinical applications (Peters et al 2001, Stinstra et al 2002, Grimm et al 2003, Lowery et al 2003).

The reduction of low and high frequency noise was more effectively performed with FastICA, reaching an average decrease of $70 \%$; being based on statistical assumptions, FastICA separates noise sources as independent signal sources that may be disregarded in the process of fetal signals retrieval, with a consequent SNR increase in fetal traces with respect to the original fMCGs. ICA has already been used in a number of studies with the specific purpose of separating noise and enhancing signals (Yoo et al 2002, Thomas et al 2002, Kobayashi et al 2002, Esposito et al 2002).

As a final remark, we can observe that, from a theoretical point of view, AMBS may produce as many fetal signals as given input traces, whereas FastICA, as all ICA algorithms, retrieves a smaller number of traces with respect to input signals. This fact can be a strong limitation for FastICA application in clinical studies using fMCG signals and requiring a precise signal source localization, such as for arrhythmogenic substrates; such localizations can be obtained with the required precision only when a detailed magnetic field mapping is available (Muller et al 1999, Tavarozzi et al 2002a, 2002b, Comani et al 2003); in that perspective it is advised to retrieve as many fetal traces as input signals. In order to achieve this target, multiple cluster configurations (Mantini et al 2004) or other approaches, using information included in the mixing matrix $A$ and presently under investigation by the authors, can be used to reproduce the original spatial distribution of fetal traces with consequent exhaustive field mapping reconstruction.

It is worth noting that signals recovered with FastICA were surely representative of fetal cardiac events without being modified by the positions of the magnetic sensors with respect to the fetal heart, as usually happens for traces reconstructed with AMBS (Stinstra et al 2002, Burghoff et al 1997); a clear example of coincident timing of cardiac waves in traces obtained with FastICA from clusters with opposite location has been given in figure 2 .

From the above considerations we can conclude that FastICA showed, in general, better performances than AMBS. It is our opinion that FastICA should be preferred for the reconstruction of the time course of fetal cardiac signals in the perspective of clinical applications, being able to provide reliable traces in a variety of different initial conditions, such as early gestational epochs or extremely noisy input signals.

Moreover, the retrieval of correct and precise P-QRS-T waves morphology, intensity and duration achieved with FastICA permitted the quantitative analysis of cardiac events on a beatto-beat basis (Comani et al 2004a, 2004c), and not only on averaged fetal beats as for signals 
obtained with AMBS: the possibility of estimating a series of temporal and spatial cardiac parameters at an intra-individual level, as well as the chance of monitoring and characterizing arrhythmias, is certainly an extremely important step forward in the clinical use of fMCG.

\section{References}

Abraham-Fuchs K, Härer W, Schneider S and Stefan H 1990 Pattern recognition in biomagnetic signals by spatiotemporal correlation and application to the localization of propagating neuronal activity Med. Biol. Eng. Comput. $28398-406$

Abraham-Fuchs K, Strohbach P, Härer W and Schneider S 1991 Improvement of neuromagnetic localisation by MCG artifact correction in MEG 8th Int. Conf. Biomagnetism (Münster, Germany) pp 429-30

Bell A J and Sejnovsky T J 1995 An information-maximization approach to blind separation and blind deconvolution Neural Comput. 7 1129-59

Burghoff B, Steinhoff U, Haberkorn W and Koch H 1997 Comparability of measurement results obtained with multi-SQUID-systems of different sensor configurations IEEE Trans. Appl. Supercond. 7 3465-8

Cardoso J F 1992 Iterative techniques for blind source separation using only fourth-order cumulants Proc. EUSIPCO (Brussels, Belgium) pp 739-42

Cardoso J F 1997 Infomax and maximum likelihood for source separation IEEE Lett. Signal Process. 4 112-4

Chaoui R, Bollmann R, Hoffmann H and Goldner B 1991 Fetal echocardiography: III. Fetal arrhytmias Zentralbl. Gynaekol. 113 1335-50

Comani S et al 2004a Fetal intra-cardiac intervals for different gestational epochs as evaluated from fetal magnetocardiograms Biomed. Tech. 48 150-2

Comani S et al 2004b Independent component analysis: fetal signal reconstruction from magnetocardiographic recordings Comput. Meth. Prog. Biomed. 75 163-77

Comani S, Liberati M, Gabriele E, Lagatta A, Di Luzio S and Romani G L 2004c Detection of fetal arrhythmias by means of magnetocardiography: a case report Biomed. Tech. 48 156-8

Comani S, Tartaro A, Lagatta A, Morana G, Di Luzio S and Romani G L 2003 Magnetocardiographic functional imaging and integration with 3D MRI reconstruction of the heart: preliminary results for source localization during myocardium activation Phys. Med. 19 119-30

Comon P 1994 Independent component analysis_a new concept? Signal Process. 36 287-314

Della Penna S et al 2000 Biomagnetic systems for clinical use Philos. Mag. 80 937-48

Esposito F et al 2002 Spatial independent component analysis of functional MRI time-series: to what extent do results depend on the algorithm used? Hum. Brain Mapp. 16 146-57

Fiori S 2003 Overview of independent component analysis technique with an application to synthetic aperture radar (SAR) imagery processing Neural Netw. (Special Issue on "Neural Networks for Analysis of Complex Scientific Data: Astronomy, Geology and Geophysics") 16 453-67

Fyfe D A, Meyer K B and Case C L 1993 Sonographic assessment of fetal cardiac arrhythmias Semin. Ultrasound CT MR 14 286-97

Grimm B et al 2003 Influence of intrauterine growth restriction on cardiac time intervals evaluated by fetal magnetocardiography Early Hum. Dev. 74 1-11

Horigome $\mathrm{H}$ et al 2001 Detection of cardiac hypertrophy in the fetus by approximation of the current dipole using magnetocardiography Pediatr. Res. 50 242-5

Horigome H, Takahashi M I, Asaka M, Shigemitsu S, Kandori A and Tsukada K 2000 Magnetocardiographic determination of the development changes in PQ, QRS and QT intervals in the foetus Acta Pediatr. 89 64-7

Hyvärinen A 1999 Fast and robust fixed-point algorithms for independent component analysis IEEE Trans. Neural Netw. 10 626-34

Hyvärinen A and Oja E 1997 A fast fixed point algorithm for independent component analysis Neural Comput. 9 283-92

Hyvärinen A and Oja E 2000 Independent component analysis: algorithms and applications Neural Netw. 13 411-30

Kaler C et al 2002 Fetal magnetocardiography: development of the fetal cardiac time intervals Prenat. Diagn. 22 408-14

Kandori A et al 2003 Classifying cases of fetal Wolff-Parkinson-White syndrome by estimating the accessory pathway from fetal magnetocardiograms Med. Biol. Eng. Comput. 41 33-9

Khaler C et al 2001 The application of fetal magnetocardiography (FMCG) to investigate fetal arrhythmias and congenital heart defects (CHD) Prenat. Diag. 21 176-82

Kleinman C S et al 1983 Fetal echocardiography. A tool for evaluation of in utero cardiac arrhythmias and monitoring of in utero therapy: analysis of 71 patients Am. J. Cardiol. 51 237-43 
Kobayashi K, Akiyama T, Nakahori T, Yoshinaga H and Gotman J 2002 Systematic source estimation of spikes by a combination of independent component analysis and RAP-MUSIC: I. Principles and simulation study Clin. Neurophysiol. $113713-24$

Lewis M J 2003 Review of electromagnetic source investigations of the fetal heart Med. Eng. Phys. 25 801-10

Lowery C L et al 2003 Noninvasive antepartum recording of fetal S-T segment with a newly developed 151-channel magnetic sensor system Am. J. Obstet. Gynecol. 188 1491-7

Mantini D et al 2004 Fetal tailoring of the independent component analysis to multi-channel fMCG recordings for an optimal reconstruction of the fetal cardiac signal Biomed. Tech. 48 186-8

Menendez T et al 2000 Prenatal diagnosis of QT prolongation by magnetocardiography PACE 23 1305-07

Mitra S K and Kaiser F 1993 Handbook for Digital Signal Processing (New York: Wiley)

Muller H P et al 1999 Localization of a ventricular tachycardia-focus with multichannel magnetocardiography and three-dimensional current density reconstruction J. Med. Eng. Technol. 23 108-15

Peters M et al 2001 Monitoring the fetal heart non-invasively: a review of methods J. Perinat. Med. 29 408-16

Stinstra J et al 2002 Multicentre study of fetal cardiac time intervals using magnetocardiography Br. J. Obstet. Gynecol. 109 1235-43

Stinstra J G and Peters M J 2002 The influence of fetoabdominal tissues on fetal ECGs and MCGs Arch. Physiol. Biochem. 110 165-76

St John Sutton M G et al 1984 Quantitative assessment of growth and function of the cardiac chambers in the normal human fetus: a prospective longitudinal echocardiographic study Circulation 69 645-54

Tavarozzi I et al 2002a Magnetocardiography: current status and perspectives: Part I. Physical principles and instrumentation Ital. Heart J. 3 75-85

Tavarozzi I et al 2002b Magnetocardiography: current status and perspectives: Part II. Clinical applications Ital. Heart J. 3 151-65

Taylor M J et al 2003 Non-invasive fetal electrocardiography in singleton and multiple pregnancies Br. J. Obstet. Gynaecol. 110 668-78

Thomas C G, Harshman R A and Menon R S 2002 Noise reduction in BOLD-based fMRI using component analysis Neuroimage 17 1521-37

van Leeuwen P, Hailer B, Bader W, Geissler J, Trowitzsch E and Gronemeyer D H 1999 Magnetocardiography in the diagnosis of fetal arrhythmia Br. J. Obstet. Gynaecol. 106 1200-8

Wakai R T, Lengle J M and Leuthold A C 2000a Transmission of electric and magnetic foetal cardiac signals in a case of ectopia cordis: the dominant role of the vernix caseosa Phys. Med. Biol. 45 1989-95

Wakai R T, Leuthold A C, Cripe L and Martin C B 2000b Assessment of fetal rhythm in complete congenital heart block by magnetocardiography Pacing Clin. Electrophysiol. 23 1047-50

Wakai R T, Strasburger J F, Li Z, Deal B J and Gotteiner N L 2003 Magnetocardiographic rhythm patterns at initiation and termination of fetal supraventricular tachycardia Circulation 107 307-12

Yang T N and Wang S D 1999 Robust algorithms for principal component analysis Phys. Rev. Lett. 20 927-33

Yoo S S, Gil Choi B, Han J Y and Hee Kim H 2002 Independent component analysis for the examination of dynamic contrast-enhanced breast magnetic resonance imaging data: preliminary study Invest. Radiol. 37 647-54 